\title{
Tecnura
}

\section{Medida de la eficiencia de un colector solar de placa plana, mediante un montaje experimental desarrollado con instrumentación virtual}

\section{Measurement of the efficiency of a flat plate solar collector, by means of an experimental assembly developed with virtual instrumentation}

\author{
Nelson Libardo Forero Chacón ${ }^{\circledR}$, Paul David Garzón Castañeda²
}

Fecha de recepción: 12 de Diciembre de 2019

Fecha de aceptación: 6 de Mayo de 2020

Cómo citar: Forero-Chacón., N.L. y Garzón-Castañeda., P.D. (2020). Medida de la eficiencia de un colector solar de placa plana, mediante un montaje experimental desarrollado con instrumentación virtual. Tecnura, 24(65) 109-124. DOI: 10.14483/22487638.15929

\section{Resumen}

Objetivo: Medir la eficiencia instantánea de un colector solar de placa plana, empleando un arreglo experimental configurado en instrumentación virtual.

Metodología: A partir de un montaje experimental desarrollado bajo la arquitectura de instrumentación virtual, que emplea un colector solar, módulos Compac FieldPoint de la firma National Istruments, y sistemas de adquisición de datos, diseñados a partir del lenguaje de programación gráfica LabVIEW, para medir radiación solar global, temperatura ambiente y temperatura del fluido del sistema, se obtiene la eficiencia instantánea del colector solar.

Resultados: Se reportan medidas de eficiencia de un colector solar de placa plana, en función de la temperatura reducida del sistema, de la hora del día y de la radiación solar global para días caracterizados por la época de la medición y acorde con el flujo de radiación solar global que incide sobre el colector. Conclusiones: El uso de un instrumento virtual y de dispositivos de alta rapidez y sensibilidad para la adquisición de datos permite medir los parámetros de operación y la eficiencia instantánea de un colector solar de placa plana tipo aleta, obteniendo un valor de promedio del 36,6\%.

Financiamiento: El Centro de Investigaciones y Desarrollo Científico de la Universidad Distrital Francisco José de Caldas financió el software LabVIEW, los módulos Compac FieldPoint y el sensor de radiación solar, en el marco de la realización del proyecto de investigación titulado "Desarrollo y prueba de prototipo de estación de medición de señales que dan información de variables ambientales", por el grupo FMAES.

Palabras clave: radiación solar global, colector solar, eficiencia, instrumentación virtual.

\footnotetext{
Abstract

Objective: To measure the instantaneous efficiency of a flat plate solar collector, using an experimental arrangement configured in virtual instrumentation.

Methodology: The instantaneous efficiency of the solar collector is obtained from an experimental
} 
setup developed with the virtual instrumentation architecture, which uses a solar collector, Compac FieldPoint modules of the National Istruments firm and data acquisition systems, designed from the LabVIEW graphic programming language, to measure solar radiation global, ambient temperature and system fluid temperature.

Results: Efficiency measures of a flat plate solar coIlector are reported, depending on the reduced temperature of the system, on the time of day and on global solar radiation for days characterized by the measurement time, and in accordance with the global solar radiation flow, that affects the collector.

Conclusions: The use of a virtual instrument and devices of high speed and sensitivity for the acquisition of data allows measuring its operation parameters and the instantaneous efficiency of a solar collector of flat plate, obtaining a value of $36.6 \%$.

Financing: The Research and Scientific Development Center of the Francisco José de Caldas District University financed the LabView software, the Compac FieldPoint modules and the solar radiation sensor, within the framework of the development of the research project called "prototype development and testing of signal measuring station that provides information on environmental variables", which was developed by the FMAES group.

Keywords: Global solar radiation, solar collector, efficiency, virtual instrumentation.

\section{INTRODUCCIÓN}

Ante la creciente demanda energética por parte de la sociedad actual, tanto urbana como rural, generada a raíz del incremento de la necesidad de servicios, así como del aumento en los índices de contaminación ambiental (MADS, 2019), causado por las fuentes finitas fósiles y su impacto evidenciado en el cambio climático y destrucción del ambiente, se hace prioritario, en general, el buen aprovechamiento energético y de los recursos energéticos renovables, en particular el recurso solar (Ayompe y Duffy, 2013). Estas situaciones han puesto como uno de los temas fundamentales y de mucha importancia a nivel mundial y regional, el desempeño y la eficiencia de los sistemas de captación y transformación energética (Devrim, Zafer y Olcay, 2015), en particular la de los colectores solares (Esen y Esen, 2005), toda vez y sabiendo en particular que, ante las condiciones ambientales y necesidades energéticas actuales, el recurso solar como fuente limpia, es prácticamente ilimitado respecto a los recursos fósiles, lo que garantiza abastecimiento a largo plazo.

El desempeño y la eficiencia de estos dispositivos solares dependen de varios factores que van desde sus características físicas, estructura, materiales empleados, configuración geométrica; pero también su ubicación y posicionamiento astronómico respecto a la incidencia de radiación solar, ya que en su conjunto, se optimiza la captación de energía, como se demuestra en innumerables investigaciones e informes recientes relacionados con características, aplicaciones y métodos para aumentar su eficiencia (Liqun, Yiping y Qunwu, 2019). Uno de los métodos actuales más efectivos de aumentar la eficiencia de estos dispositivos es reemplazar el fluido de trabajo, que convencionalmente ha sido agua, por fluidos que poseen alta conductividad térmica. En los últimos años, se han venido utilizando fluidos que contienen nanopartículas sólidas suspendidas en un fluido base y denominados nanofluidos, los que, se estima, presentan propiedades de transferencia de energía térmica en forma excepcional, comparados con fluidos de transferencia convencionales. En lo que conlleva al interés del presente trabajo, no puede dejarse tampoco, de lado, la capacidad y versatilidad de los sistemas de medición que permite el desarrollo tecnológico actual y como lo es la instrumentación virtual (Vestlund, Dalenba y Ronnelid, 2012), ya que cumple con precisión las 
medidas y el continuo monitoreo de parámetros ambientales y de operación de dichos sistemas solares. En este trabajo se da evidencia de las ventajas y alcances de un desarrollo experimental con instrumentación virtual, que permite el monitoreo continuo del desempeño de un sistema colector solar, con la medida de su eficiencia instantánea. Se reportan medidas de eficiencia de un colector solar de placa plana (tipo aleta), en función de la temperatura reducida del sistema, de la hora del día y de la radiación solar global para unos días en particular, caracterizados por la época del año y acorde con el flujo de radiación solar global incidente $\mathrm{G}_{\mathrm{i}}$, con ayuda de sistemas de adquisición de datos vinculados a la estación de monitoreo de parámetros ambientales y atmosféricos del grupo de investigación en Física del Medio Ambiente y Energía Solar (FMAES), estación ubicada a 4,61 de latitud norte $74,06^{\circ}$ de longitud oeste y a una altitud de 2657 msnm, sede Macarena B de la Universidad Distrital Francisco José de Caldas en Bogotá, Colombia.

\section{METODOLOGÍA}

\section{Aspectos conceptuales y experimentales}

Un colector solar transforma la energía solar global $G_{i}$, que incide sobre la placa en forma de radiación electromagnética en energía térmica (calor) útil $\mathrm{q}_{\text {util' }}$ transmitiéndola por conducción a un sistema de circulación de fluido que, a su vez, es calentado por convección (Duffie y Beckman, 2013). De esta forma es posible plantear una ecuación de balance energético entre el calor absorbido por la placa y el disipado en los procesos de convección y conducción; así es posible hallar el flujo de calor transmitido por convección en cualquier instante (o calor útil) por la superficie radiante. Existen diversos métodos para probar el rendimiento de colectores solares de diversos tipos, siendo de los más utilizados a nivel mundial, los establecidos por la Ashrae, (ASHRAE, 2003). La eficiencia $\eta$ para el colector solar de placa plana (denominado tipo de aleta), se define a través de la ecuación (1), como:

$$
\eta=\frac{\mathrm{q}_{\text {util }}}{\mathrm{q}_{\text {incidente }}}
$$

Para esto, se establece el balance de energía del dispositivo solar, en el que se iguala la energía incidente sobre el colector dada por $\mathrm{G}_{\mathrm{i}} \mathrm{A}_{\mathrm{c}}(\tau \alpha)$, con el calor útil $q_{\text {util }}$ las pérdidas totales de energía $\left(q_{\text {perd }}\right)$ y la energía almacenada por el mismo colector dU/dt. La ecuación (2), expresa todo así:

$$
G_{i} A_{c}(\tau \alpha)=\mathrm{q}_{\mathrm{util}}+\mathrm{q}_{\mathrm{perd}}+\frac{d U}{d t}
$$

Donde: $\mathrm{A}_{\mathrm{c}}$ es el área del colector y el producto $\tau \alpha$ corresponde al coeficiente producto de transmitancia-absorbancia con incidencia normal de radiación sobre la placa.

Las pérdidas de energía (calor) están dadas, como:

$$
q_{\text {perd }}=U_{L} A_{c}\left(T_{p}-T_{a}\right)
$$

Donde: $U_{L}$ es el coeficiente de pérdidas globales del colector, $A_{C}$ es el área del colector, $T_{p}$ es la temperatura de la placa y $\mathrm{T}_{\mathrm{a}}$ es la temperatura ambiente. Si se asume dentro del balance de energía que $\mathrm{dU} / \mathrm{dt}$ tiende a cero cuando la temperatura interna del sistema es igual a la temperatura ambiente, y el sistema tiene el tiempo suficiente para que el calor útil corresponda a la energía que incide (constante de tiempo del sistema), y se aplican condiciones de frontera, obteniendo como parámetros de temperatura, la temperatura ambiente $\mathrm{T}_{\mathrm{a}^{\prime}}$ la temperatura del fluido a la entrada del colector $\mathrm{T}_{f}$ la masa de agua que fluye por el colector $\dot{m}$, el calor específico del agua $\left(C_{p}\right)$ y el coeficiente de pérdidas globales $\left(U_{L}\right)$, entonces la eficiencia instantánea para el colector solar, tipo aleta, expresada en la ecuación (1), da como resultado:

$$
\eta=\frac{q_{u t i l}}{\mathrm{q}_{\text {incidente }}}=\frac{\dot{m} C_{P} \Delta T}{G_{i} A_{c}}=F_{r}(\tau \alpha)-\frac{F_{r} U_{L}}{10} T_{R}
$$


En la ecuación (4), el factor $T_{R}$ es la temperatura reducida del sistema, dada por:

$$
T_{R}=10 \mathrm{~W} / \mathrm{m}^{2}\left(\frac{T_{f}-T_{a}}{G_{i}}\right)
$$

El término compuesto $F_{r}(\tau \alpha)$ es la fracción de irradiación que se trasmite a través de las cubiertas, el cual no incrementa la energía interna de la placa colectora y cuyo resultado es conocido como eficiencia óptica del dispositivo, valor que se obtiene cuando $\mathrm{T}_{\mathrm{a}}$ y $\mathrm{T}_{\mathrm{f}}$ son iguales. El producto $(\tau \alpha)$ corresponde al producto entre los coeficientes de transmitancia-absorbancia.

El factor $F_{r}$ en la ecuación (4), es conocido como factor de remoción y está dado por:

$$
F_{r}=\frac{\dot{m} C_{P}}{A_{c} U_{L}}\left[1-e^{\left[-\frac{A_{c} U_{L} F^{\prime}}{m \dot{C}_{P}}\right]}\right]
$$

Aquí, $\mathrm{F}^{\prime}$ es el factor de eficiencia del colector, expresado a continuación en la ecuación (7) como:

$$
F^{\prime}=\frac{1 / U_{L}}{W\left[\frac{1}{U_{L}[D+(W-D) F]}+\frac{1}{C_{b}}+\frac{1}{\pi D_{i} h_{f i}}\right]}
$$

Al utilizar el concepto de eficiencia de aleta, el resultado para la eficiencia estándar de aleta, para aletas rectas que se presenta en la ecuación (7) como el factor $F$, se puede expresar como:

$$
F=\frac{\tanh [m(W-D) / 2]}{m(W-D) / 2}
$$

Este es el factor de aleta. En las ecuaciones (7) y (8), $D_{i}$ es el diámetro interno del tubo; $C_{b^{\prime}}$ la conductancia de la soldadura; $m$ es un parámetro que reúne a $U_{L}$ y otras constantes; $h_{f i}$ es el coeficiente de transferencia de calor entre el fluido y la pared del tubo por convección; $W$ es la distancia entre los tubos del colector, y $\mathrm{D}$ es el diámetro exterior de cada tubo.

La tabla 1 presenta los parámetros técnicos estándar del colector solar utilizado, así como valores de las medidas directamente hechas sobre el arreglo experimental y empleados en la ecuación (4). Así, la función de eficiencia obtenida,está por:

$$
\eta=0.692-0.457 \mathrm{~T}_{R}
$$

La ecuación (9) representa la esencia del experimento, a partir de la cual y con el sistema de adquisición de datos desarrollado, esto es, el instrumento virtual IV, se calcula la eficiencia instantánea del colector de placa plana tipo aleta, en función de la temperatura reducida (Álvarez, Cabeza, Muñiz y Valera, 2010), con la que a su vez se determina el rendimiento en el tiempo y se obtienen las curvas características de eficiencia vs. temperatura reducida, y eficiencia vs. radiación solar incidente, y de estas se determina la eficiencia instantánea del dispositivo, que fue uno de los propósitos del estudio.

Tabla 1. Parámetros utilizados para medir la eficiencia instantánea en el colector utilizado

\begin{tabular}{ll}
\hline \multicolumn{1}{c}{$\begin{array}{c}\text { Masa de agua que fluye al colector } \\
(\boldsymbol{m})\end{array}$} & \multicolumn{1}{c}{$\mathbf{0 , 0 1 0} \mathbf{~ k g} / \mathbf{s}$} \\
\hline Calor específico del agua $\left(\mathrm{C}_{\mathrm{p}}\right)$ & $4,18 \times 10^{3} \mathrm{~J} / \mathrm{kgK}$ \\
\hline Coeficiente de pérdidas globales $\left(\mathrm{U}_{\mathrm{\perp}}\right)$ & $5,14 \mathrm{~W} / \mathrm{m}^{2} \mathrm{~K}$ \\
\hline Área del colector $\left(\mathrm{A}_{\mathrm{c}}\right)$ & $1,22 \mathrm{~m}^{2}$ \\
\hline Distancia entre tubos $(\mathrm{W})$ & $0,100 \mathrm{~m}$ \\
\hline Diámetro externo de las tuberías $(\mathrm{D})$ & $0,015 \mathrm{~m}$ \\
\hline Diámetro interno de las tuberías $\left(\mathrm{D}_{\mathrm{i}}\right)$ & $0,014 \mathrm{~m}$ \\
\hline Conductancia de la soldadura $\left(\mathrm{C}_{\mathrm{b}}\right)$ & $58,0 \mathrm{~W} / \mathrm{m}^{2} \mathrm{~K}$ \\
\hline Conductancia de la placa $(\mathrm{K})$ & $51,7 \mathrm{~W} / \mathrm{mK}$ \\
\hline Espesor de la placa $(\delta)$ & $6 \times 10^{-4} \mathrm{~m}$ \\
\hline $\begin{array}{l}\text { Coeficiente de película entre las tuberías y } \\
\text { el agua }\left(\mathrm{h}_{\mathrm{fi}}\right)\end{array}$ & $300 \mathrm{~W} / \mathrm{m}^{2} \mathrm{~K}$ \\
\hline Transmitancia a través de las cubiertas $(\tau)$ & 0,875 \\
\hline $\begin{array}{l}\text { Absorción de radiación de la placa colec- } \\
\text { tora }(\alpha)\end{array}$ & 0,890 \\
\hline
\end{tabular}

Fuente: cálculos y mediciones propias, algunas son valores estándar.

\section{Descripción de la configuración experimental. Instrumento virtual}

Un colector solar es un intercambiador de calor tipo aleta finita, que captura energía útil de la radiación solar global $\mathrm{G}_{\mathrm{i}}$ que incide sobre la placa colectora del sistema y que presenta un margen de 
pérdidas de energía típico de un sistema no ideal. La medición y monitoreo de los parámetros a medir para determinar la eficiencia del colector solar son la irradiación solar global $\mathrm{G}_{\mathrm{i}}$ la temperatura a la entrada del fluido $T_{f}$ y la temperatura ambiente $T_{a}$.

En la figura 1, se representa el diagrama de bloques general del montaje experimental, para la medida de la eficiencia instantánea del colector solar, en conjunto con el diagrama de bloques del instrumento virtual desarrollado.

La medición y monitoreo se realizan a partir de sistemas de adquisición de datos diseñados bajo el concepto de instrumento virtual (IV), mediante el lenguaje de programación gráfica $L a b$ VIEW, que emplea como componentes hardware, módulos compact Field Point (cFP), computador, sensores y dispositivos. Tanto LabVIEW, como los
cFP son desarrollados por la firma National Instruments. Los cFP están compuestos por un módulo compact Field Point-2020 (cFP-2020), que es un controlador de procesos de automatización programable y módulos I/O, cFP AI 100 y cFP TC 120, estos últimos para medir diferentes variables (señales) provenientes de los sensores utilizados. Las comunicaciones entre el sistema cFP-2020 y el PC se efectúan a través de puerto Ethernet y la configuración de los diferentes dispositivos es llevada a cabo con el software Measurement \& Automation Explorer $(M A X)$, desarrollado por National Instruments. Los datos capturados siguen la estructura, secuencia y procesamiento establecidos a partir de cada instrumento virtual diseñado abiertamente en LabVIEW. En su caso, el subinstrumento virtual (sublV) diseñado para la medida de la

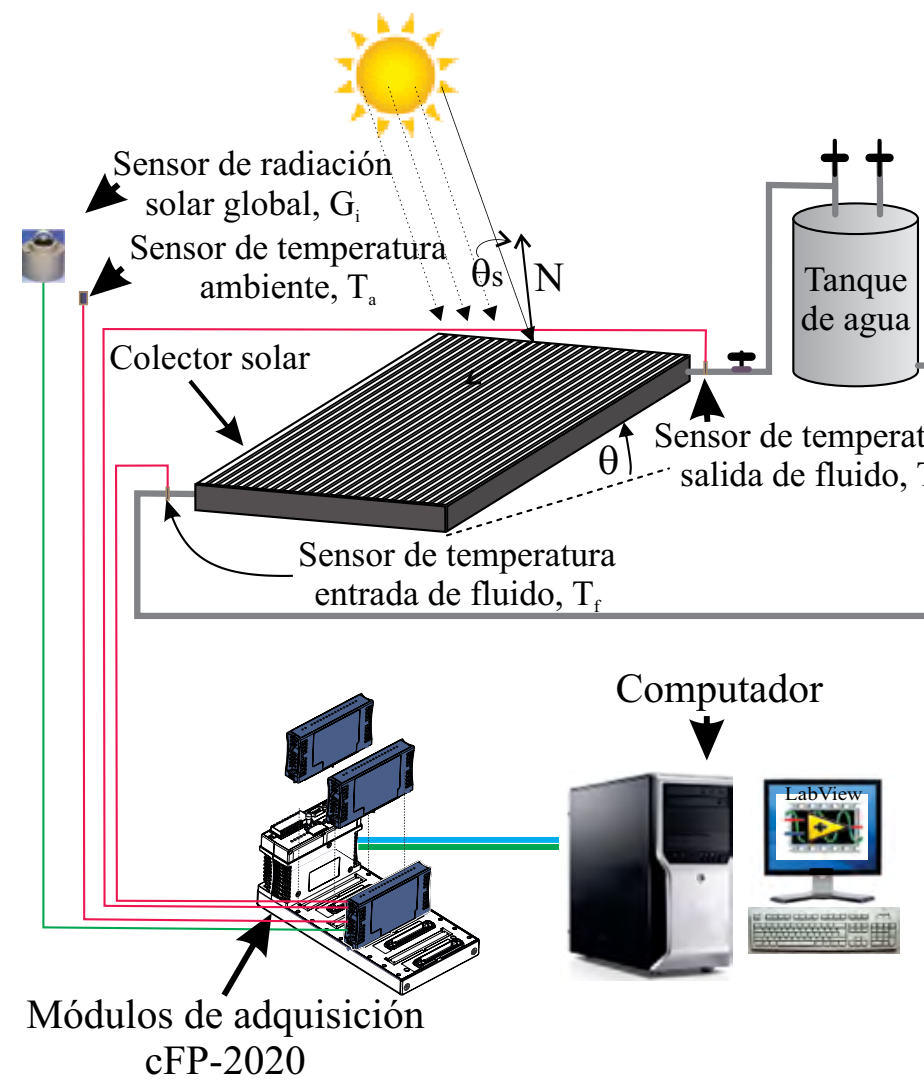

Figura 1. Diagrama de bloques del montaje experimental para la medida de la eficiencia instantánea de un colector solar e instrumento virtual

Fuente: elaboración propia. 
radiación solar global G la cual incide sobre el colector, es adquirida a partir de un sensor de radiación global, que es un piranómetro tipo termopila de la firma Eppley ref. 8-48, Black \& White nivelado horizontalmente, con sensibilidad de $10 \mu \mathrm{V} /$ $\mathrm{Wm}^{-2}$ y tiempo de respuesta del orden de 4,2 segundos. En conjunto, el sublV tiene una resolución de $\pm 1,2 \mathrm{Wm}^{-2}$ y permite visualizar los resultados en una interfaz gráfica denominada panel frontal, como se muestra en las figuras 2 y 3 .
En forma análoga, se desarrollaron subIV para las medidas de las temperaturas de entrada del fluido $T_{f}$ y temperatura ambiente $T_{a}$ y emplean como sensores de temperatura, en cada caso, un termistor NTC de 5,0 KOhm. Estos sensores poseen un rango de operación entre 233,16 K a $573,16 \mathrm{~K}$, con un error de $\pm 0,1 \mathrm{~K}$ en un rango de 273,16 K a 323,16 K. La resolución de todo el IV para la medida de temperatura en escala centígrada está dada por $\pm 0,1^{\circ} \mathrm{C}$. Cada 5 segundos el IV

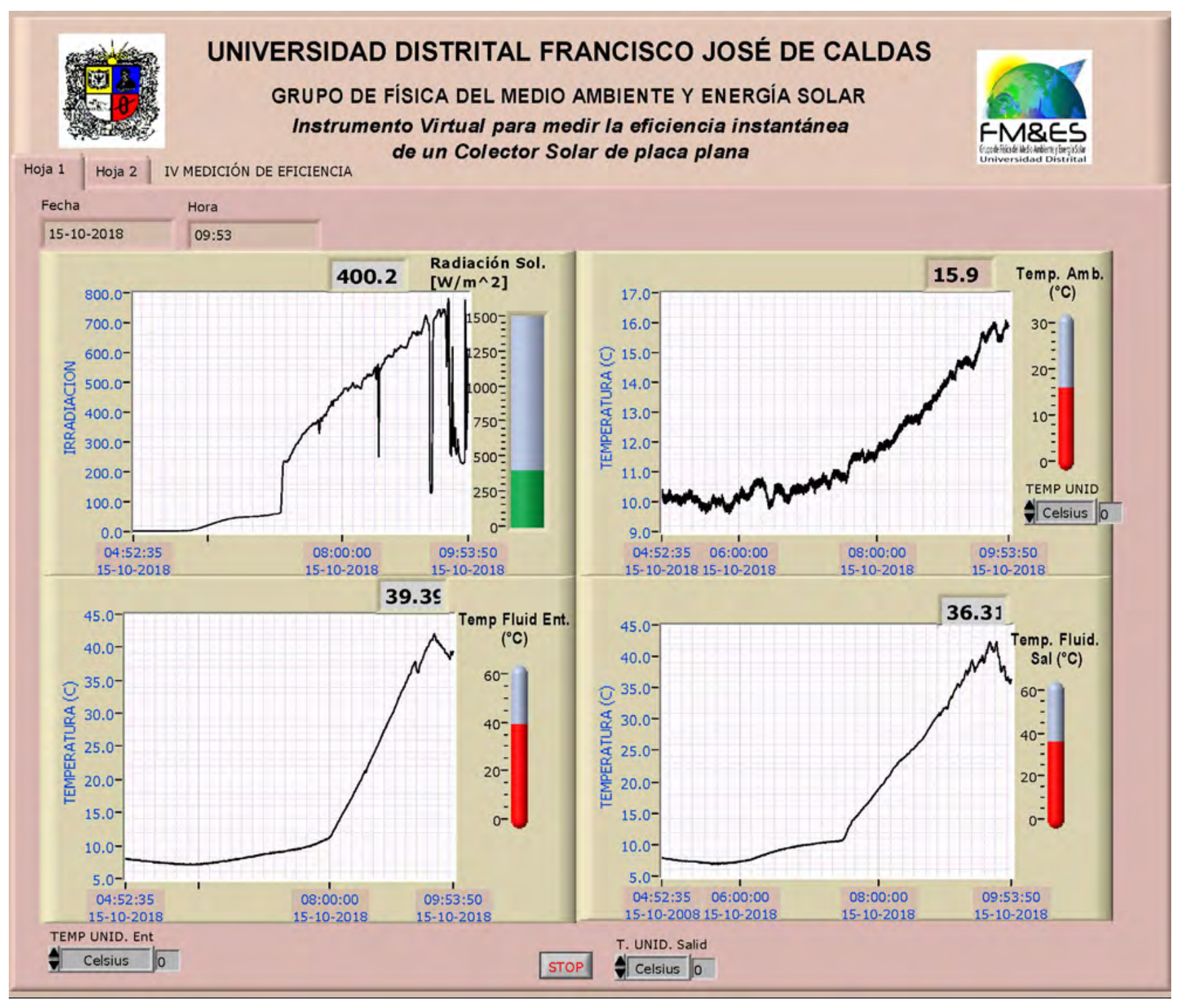

Figura 2. Panel frontal del IV que mide la eficiencia instantánea (hoja 1)

Fuente: elaboración propia a partir de LabVIEW. 
realiza una adquisición de las señales de entrada en forma simultáneamente para cada parámetro a medir, el cual se transduce y transforma matemáticamente según el mecanismo o fenómeno físico que lo rige y genera el valor de la medida. EI IV ejecuta 12 adquisiciones consecutivas y calcula el promedio, esto es el valor del promedio de la medida por minuto, luego lo exporta y guarda, en un archivo "dd-mm-AAA.xIsx" por día para generar la base de datos.

\section{RESULTADOS}

Uno de los resultados del arreglo experimental es el desarrollo del IV, el cual y a manera de ejemplo, la figura 2, representa su panel frontal y que contiene dos hojas o páginas. La primera de ellas presenta luego de la ejecución de los procesos matemáticos necesarios dentro de la estructura y configuración que se realiza en el diagrama de conexiones del instrumento, los valores de las medidas de las variables de entrada al sistema, esto es, la radiación solar global, la temperatura ambiente, la temperatura de entrada del fluido al colector y la temperatura de salida, tomando en cuenta la hora y día de registro de la medición.

En la hoja 2 del panel frontal, y simultáneamente al proceso de adquisición de las medidas de las variables de entrada visualizadas en la hoja 1, el instrumento virtual mediante la estructuración de los procesos matemáticos correspondientes ejecuta los cálculos y determina los valores de la temperatura reducida y la eficiencia instantánea cada 5 segundos, obteniendo igualmente con las doce adquisiciones, los promedios minuto de estos dos parámetros y se presentan sus valores, como se muestra en la figura 3 a manera de ejemplo la imagen de la hoja 2 del panel frontal del IV.

En esta página se revela la evolución de la eficiencia instantánea en función de la hora del día (del tiempo). Estas observaciones son necesarias para hacer seguimiento del desempeño y comportamiento del colector durante las horas de sol (desde las 6:00 hasta las 18:00). A partir de allí el IV

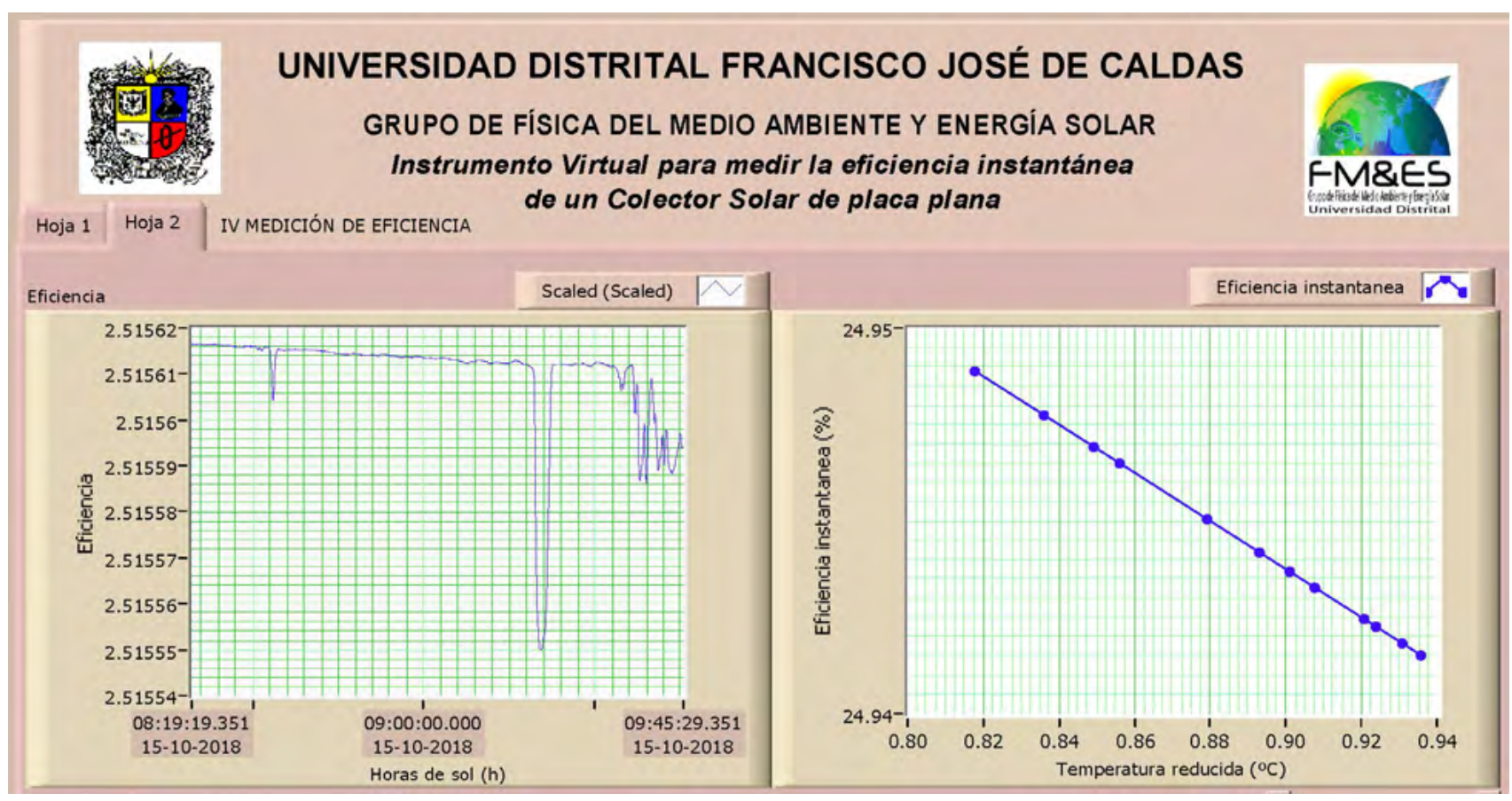

Figura 3. Panel frontal del IV que mide la eficiencia instantánea (hoja 2)

Fuente: elaboración propia a partir de LabVIEW. 
arroja los valores estadísticos de las eficiencias mínima, máxima y media, e igualmente los valores correspondientes de la temperatura reducida del sistema. Los datos anteriores son salvados en bases de datos para la eficiencia y la temperatura reducida, ambas en función de la hora del día, con el fin de llevar a cabo el análisis del comportamiento del colector.

De otra parte, el diagrama de conexiones del IV en el que se estructuran cada una de sus funciones y cuyos resultados se visualizan en el panel frontal, se presenta en la figura 4. Cada ícono en particular representa y desempeña operaciones, funciones o tareas específicas, ligadas a través de líneas que a su vez representan el flujo de información y procesamiento de las señales (datos) y que de acuerdo con la secuencia fluyen de izquierda a derecha en dicho diagrama.

Con ayuda del IV desarrollado se obtiene la eficiencia de aleta del colector solar de placa plana, permitiendo a su vez calcular la eficiencia instantánea ( $\eta$ ) del dispositivo, su desempeño en el tiempo y las curvas características: eficiencia contra temperatura reducida, eficiencia contra radiación incidente y eficiencia contra hora del día.

\section{Análisis de resultados}

Para establecer un panorama general del comportamiento de la eficiencia del colector solar y verificar el desempeño del IV desarrollado para determinarla, se han establecido tres rangos de $\mathrm{G}_{\mathrm{i}}$, siguiendo los criterios para las características del día, conforme a otras investigaciones del grupo FMAES (Forero, Caicedo y Gordillo, 2007):
- Días nublados:

$$
0<\overline{G_{l}} \leq 200 \mathrm{~W} / \mathrm{m}^{2} \text {. }
$$

- Días parcialmente nublados:

$$
200 \mathrm{~W} / \mathrm{m}^{2}<\overline{G_{\iota}} \leq 400 \mathrm{~W} / \mathrm{m}^{2} \text {. }
$$

- Días claros:

$$
400 \mathrm{~W} / \mathrm{m}^{2}<\overline{G_{l}} .
$$

En la tabla 2, se presentan los resultados de los promedios para tres días característicos tomados al azar y siguiendo los criterios señalados anteriormente y a manera de ejemplo, de la irradiación promedio diaria $\bar{G}_{l}$, la eficiencia instantánea promedio $\bar{H}_{\iota}$ y la temperatura máxima del agua a la salida del colector $\mathrm{T}_{\text {s-máx }}$.

Lasfiguras 5, 6 y 7 representan el comportamiento y seguimiento de las medidas de radiación solar global (izquierda), temperatura ambiente y de temperatura de entrada del fluido (derecha), y desempeño del colector de placa plana, tipo aleta, conforme al montaje experimental desarrollado, para cada día característico establecido a partir de los criterios señalados en la tabla 2.

Al observar los resultados representados en las figuras 5, 6 y 7 se evidencia el seguimiento que hace la temperatura de entrada del fluido $T_{f}$ (en color verde), al comportamiento de la radiación solar global $\mathrm{G}_{\mathrm{i}}$, con máximos coincidentes en el tiempo para ambas curvas entre las 12:00 y 13:30 horas, particularmente. La temperatura del fluido crece con rapidez y casi a la misma tasa en que la irradiación se incrementa, por tanto, cualquier cambio de la magnitud de la radiación genera cambios significativos en la temperatura del

Tabla 2. Días característicos, irradiación y eficiencia instantánea promedio y temperatura máxima del agua $\mathrm{T}_{\text {s-máx }}$

\begin{tabular}{lccc}
\hline \multicolumn{1}{c}{ Día característico } & $\bar{G}_{\boldsymbol{\imath}}\left(\mathbf{W m}^{-\mathbf{2}}\right)$ & $\mathbf{T}_{\text {máx }}\left({ }^{\circ} \mathbf{C}\right)$ & $\bar{H}_{\iota}(\mathbf{\%})$ \\
\hline Nublado & 188,1 & 41,2 & 55,7 \\
\hline Parcialmente nublado & 370,0 & 65,6 & 48,3 \\
\hline Claro & 429,2 & 75,0 & 55,6 \\
\hline
\end{tabular}

Fuente: elaboración propia. 
Medida de la eficiencia de un colector solar de placa plana

Forero-Chacón., N.L. y Garzón-Castañeda., P.D.

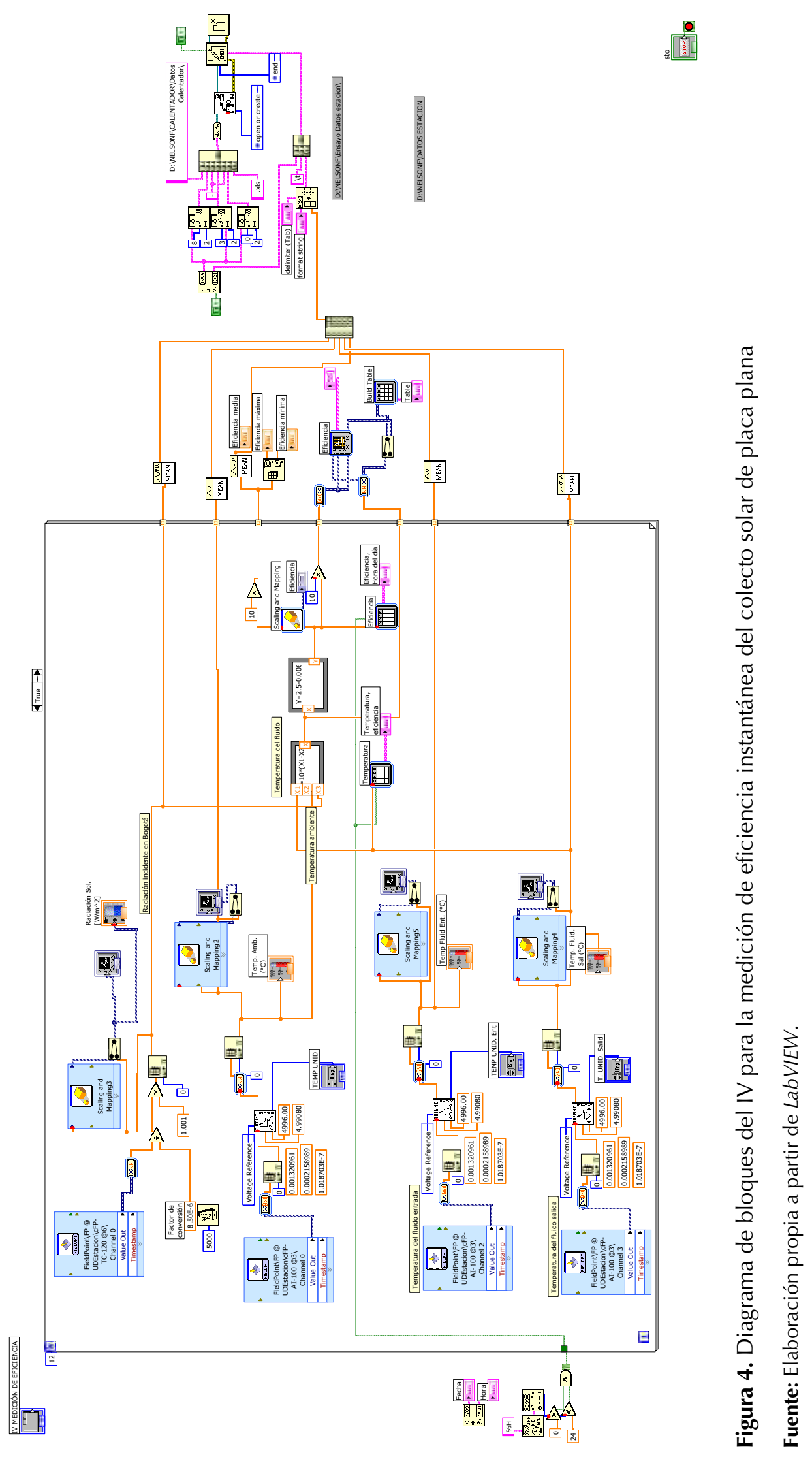

Tecnura • p-ISSN: 0123-921X • e-ISSN: 2248-7638 • Vol. 24 No. $65 \bullet$ Julio - Septiembre de $2020 \bullet$ pp. 109-124

[ 110 ] 
fluido $T_{f}$, demostrando que esta última caracteriza a su vez, el comportamiento de la radiación solar (o viceversa). En contraste, es menos significativo el seguimiento de la temperatura ambiente $\mathrm{T}_{\mathrm{a}^{\prime}}$ a los cambios de la radiación solar y, por tal razón, la dependencia que puede tener la temperatura del fluido $T_{f}$ de la temperatura ambiente $T_{a^{\prime}}$ es baja, a pesar de que ambas alcanzan su máximo valor por lo general en la misma franja horaria. Un argumento al respecto de este comportamiento es que la temperatura ambiente, en aproximación, es constante bajo el mismo régimen de radiación solar. Nótese que, por ejemplo, para el día parcialmente nublado, el punto máximo de temperatura del
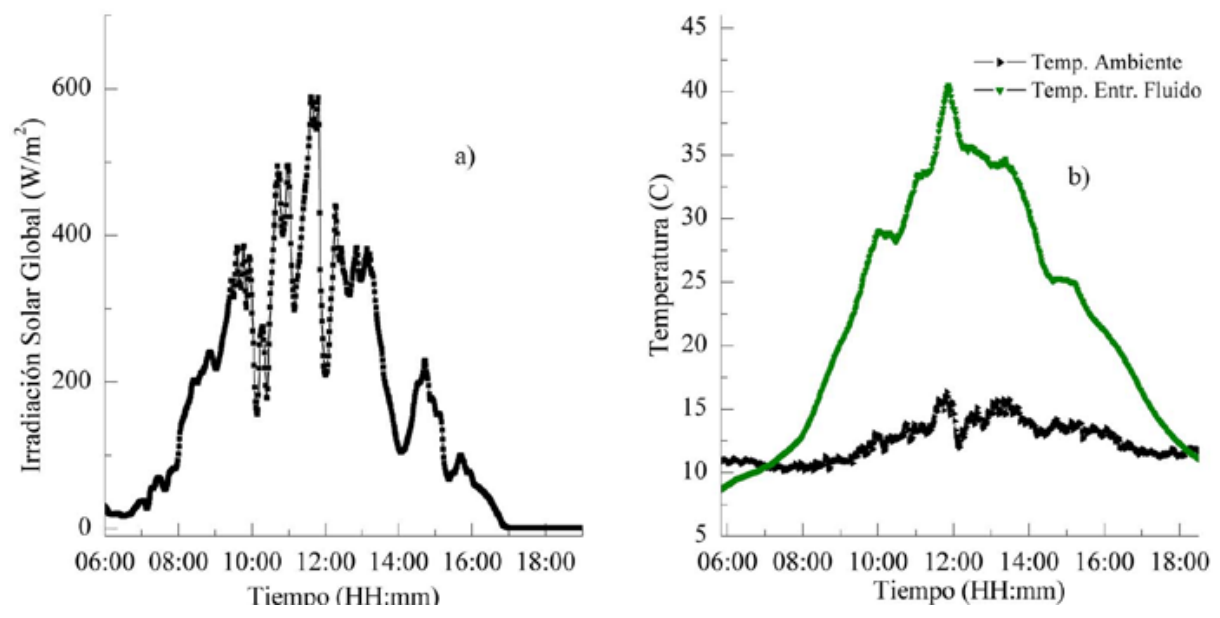

Se presenta en a) radiación solar global $G_{i}$ y en b) temperatura ambiente $T_{a}$ y temperatura entrada del fluido $T_{s}$.

Figura 5. Medidas a partir del IV desarrollado de las variables de entrada para determinar la eficiencia de un colector solar de placa plana tipo aleta, para un día caracterizado como nublado

Fuente: elaboración propia.
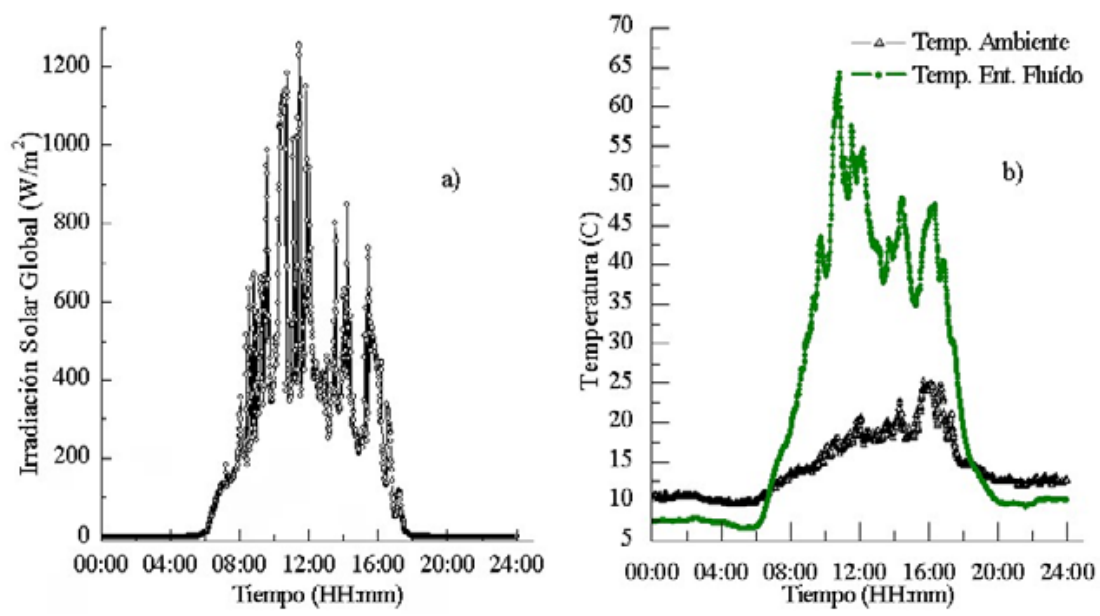

Se muestra en a) radiación solar global $G_{i}$ y b) temperatura ambiente $T_{a}$ y temperatura entrada del fluido $T_{f}$.

Figura 6. Medidas de variables de entrada para determinar la eficiencia de un colector solar de placa plana, durante un día caracterizado como parcialmente nublado

Fuente: elaboración propia. 
fluido se obtiene cerca de las 11:00 horas, cuando la temperatura ambiente $T_{a^{\prime}}$ aún y con antelación de varias horas, no alcanza su máximo. Con esto se ratifica que el calentamiento del fluido se debe, en mayor proporción a la radiación solar que incide.

Para complementar el análisis sobre los resultados descritos anteriormente en torno al comportamiento térmico del fluido, frente a la incidencia de radiación solar global en el colector, es necesario determinar la eficiencia de este, y analizar su comportamiento frente a la incidencia de radiación solar. Las figuras 8, 9 y 10 muestran los resultados de las medidas de la eficiencia del colector en función de la radiación solar global (izquierda) y de la eficiencia en función
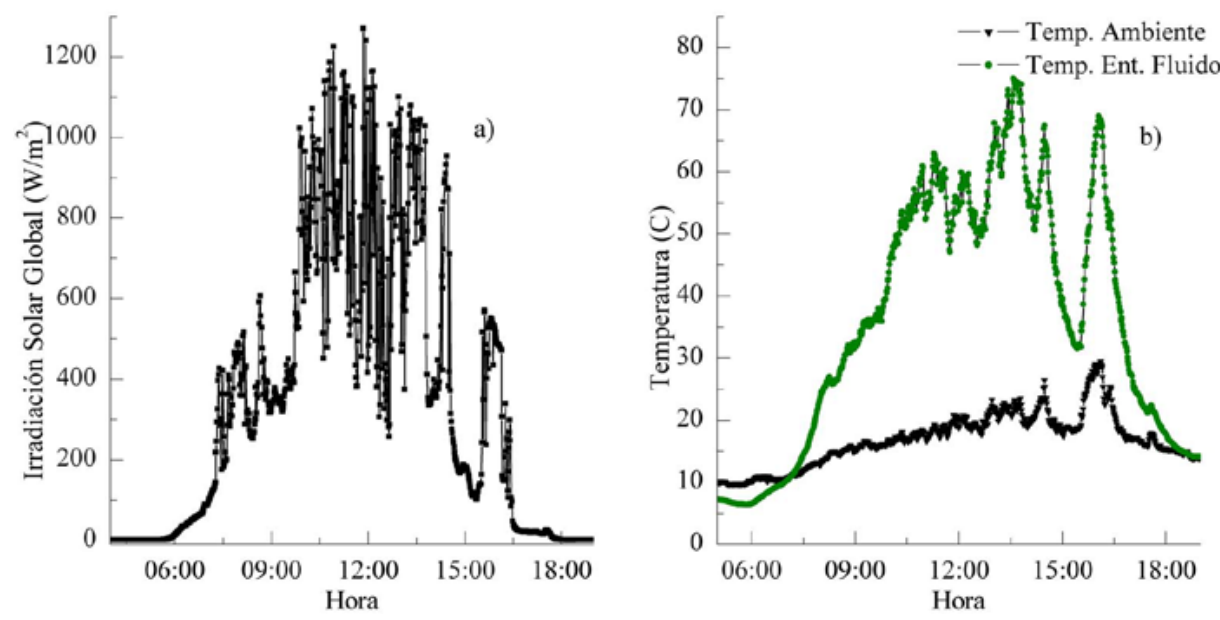

a) Radiación solar global $G_{i}$ y b) temperatura ambiente $T_{a}$ y temperatura entrada del fluido $T_{f}$.

Figura 7. Medidas de las variables de entrada para determinar la eficiencia de un colector solar de placa plana, durante un día caracterizado como claro

Fuente: elaboración propia.
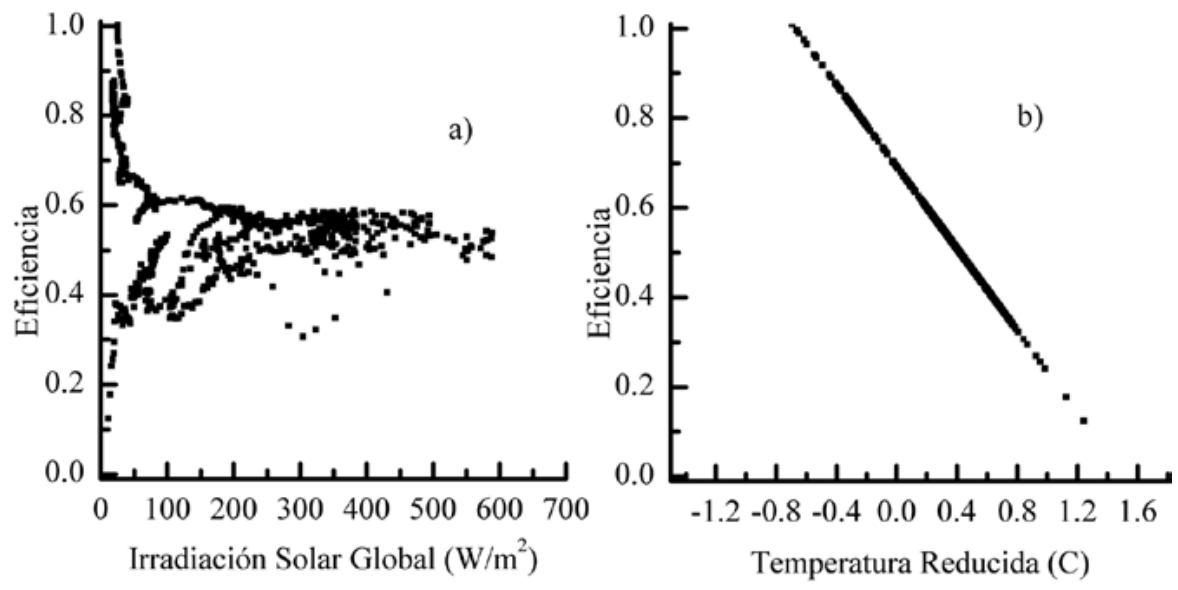

Figura 8. Eficiencia del colector solar correlacionada en función de a) irradiación solar global $G_{i}$ y b) temperatura ambiente para un día caracterizado nublado

Fuente: elaboración propia. 
de la temperatura reducida del sistema $T_{R}$ (derecha), para los mismos días característicos, citados en el apartado anterior.

Es de notar, para estos días caracterizados como nublados y parcialmente nublados, que el resultado de correlacionar la eficiencia $\eta$ del colector con la temperatura reducida del sistema $T_{R^{\prime}}$ da positiva, en contraste con los resultados para días de alta radiación, esto es claros, en los que se obtienen valores negativos, como muestra la figura 10.

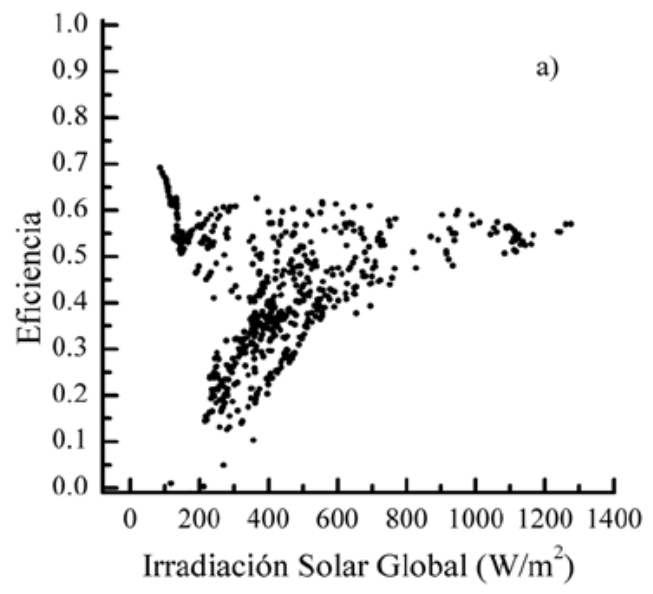

Se observa que, cuando la radiación es baja, en particular en las primeras horas de sol en la mañana, se obtienen valores elevados de eficiencia $\eta$. Este comportamiento se explica, al considerar que, en dichas franjas horarias, la temperatura del fluido es baja, por lo que el régimen de transferencia de energía radiante a través del sistema al fluido es mayor, incrementando rápidamente la temperatura de este.

Al aumentar la intensidad de radiación solar que incide en el colector, la eficiencia tiende a

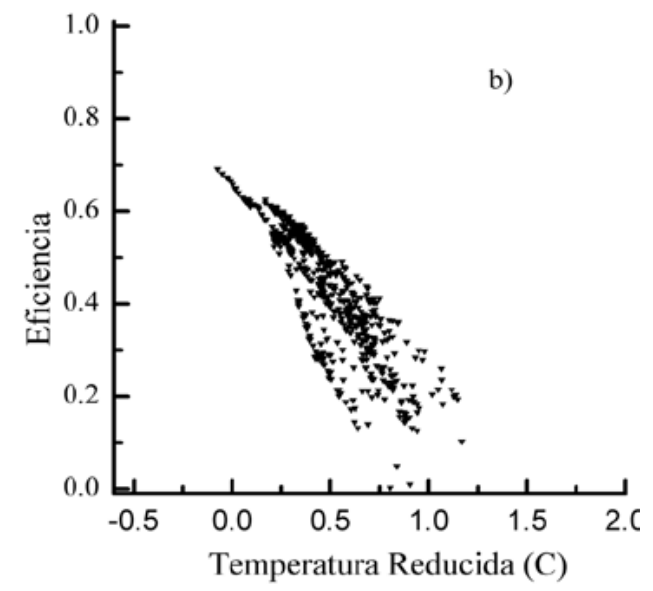

Figura 9. Eficiencia del colector solar de placa plana en correlación con: a) irradiación solar global $\mathrm{G}_{\mathrm{i}}$ y b) temperatura reducida, para un día caracterizado como parcialmente nublado

Fuente: elaboración propia.
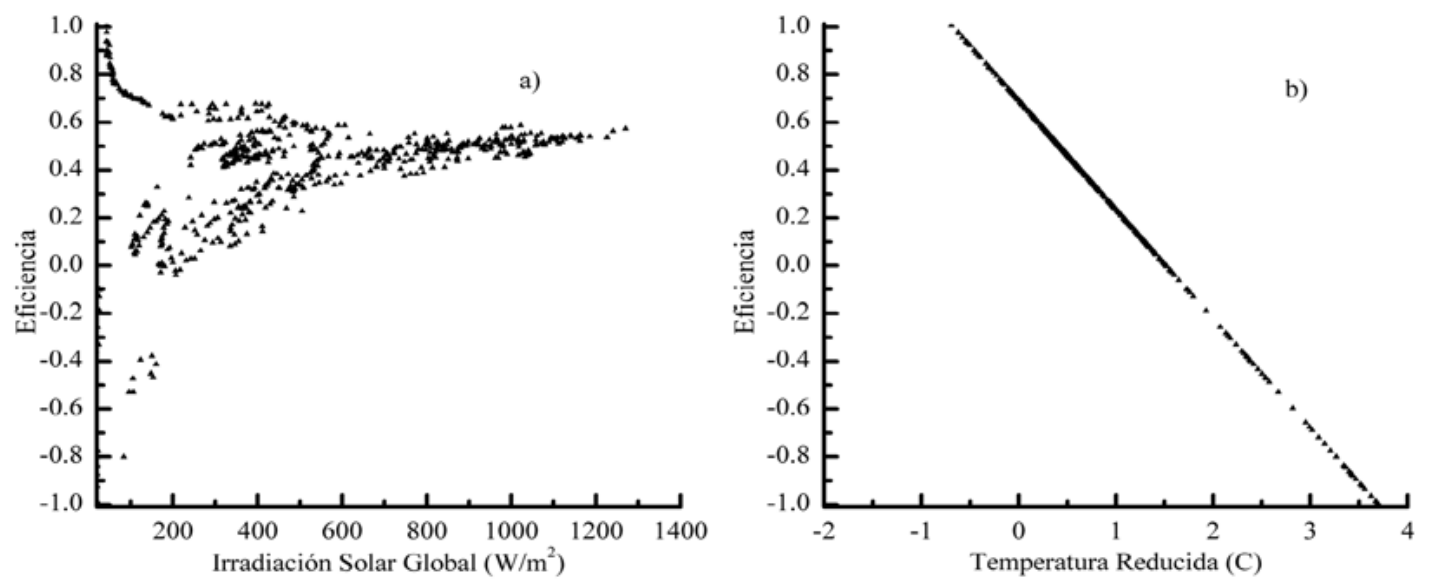

Figura 10. Eficiencia del colector solar de placa plana en correlación con: a) irradiación solar global y b) temperatura reducida, para un día caracterizado como claro

Fuente: elaboración propia. 
mantenerse en una banda de valor constante, salvo por picos mínimos en el día nublado y en el día claro atribuidos a procesos de transferencia de energía en sentido contrario, esto es, en el que la energía se transfiere del fluido a la placa del colector. La banda ancha que se presenta en la correlación de la eficiencia $\eta$ en función de la radiación solar global $\mathrm{G}_{\mathrm{i}}$ para el día parcialmente nublado, se atribuye a las abundantes fluctuaciones de la radiación solar sobre el dispositivo, causadas por la presencia intermitente de nubes. Puede observarse que esos picos en sentido negativo son pocos en comparación con la franja de eficiencia promedio del sistema, esto significa que el régimen de trabajo del dispositivo permanece relativamente constante, de tal forma que el fluido siempre incrementa su temperatura hasta estabilizarse, a pesar de ser un día nublado o de baja radiación solar. Se evidencia que la eficiencia del dispositivo es mayor cuando la radiación solar permanece estable, situación que en particular se presenta en días totalmente nublados, o en su defecto, días totalmente claros.

Los resultados del comportamiento de la correlación de la eficiencia $\eta$ en función de la temperatura reducida $T_{R^{\prime}}$ satisface lo reportado en la literatura, en cuanto a la tendencia lineal con pendiente negativa (Saedodin et al., 2017). Es notable que existan instantes en los que la eficiencia instantánea es mayor al promedio (esto es, por encima del $60 \%$ ). No significa la inexistencia de pérdidas de energía, sino que el dispositivo transfiere energía en forma óptima al sistema, como consecuencia de la estabilidad de las condiciones climáticas. La pendiente negativa de la recta muestra que la eficiencia instantánea decae mientras la temperatura reducida aumenta, situación que se da cuando la temperatura del fluido es mayor a la del sistema colector, lo que significa que el fluido transfiere (cede) más energía al sistema de la que absorbe de él.

Otra forma de estudiar la eficiencia del colector es evidenciar su comportamiento en función de la hora del día, conforme al comportamiento de la radiación solar y de las condiciones de temperatura ambiente. La figura 11 muestra las curvas de eficiencia $\eta$ en función de la hora del día, para un día caracterizado como nublado.

Se observa el comportamiento de la eficiencia sobre las 6:00 horas con valores altos, cercanos al $80 \%$, mientras que disminuye a medida que transcurre el día y se hace mínima, pasadas las 16:00 horas. Análogamente, en la figura 12, se presenta la curva de eficiencia $\eta$ en función de

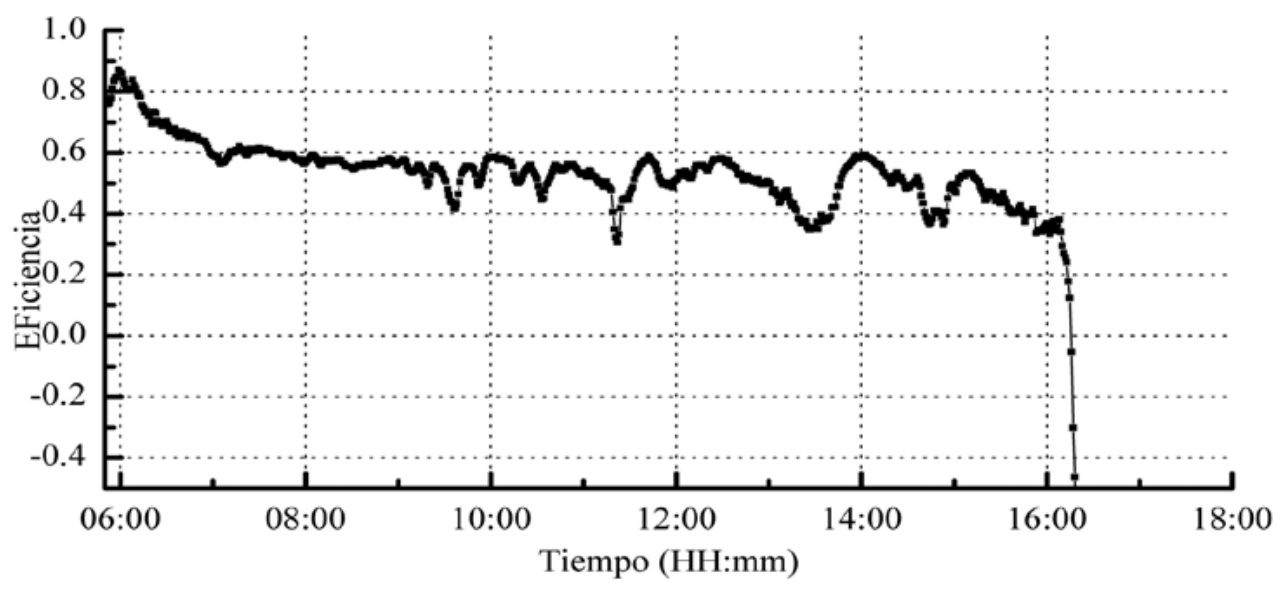

Figura 11. Comportamiento de la eficiencia $\eta$ del colector solar de placa plana tipo aleta con respecto al tiempo, para un día caracterizado como nublado

Fuente: elaboración propia. 
la hora del día, para un día caracterizado como parcialmente nublado.

Para días caracterizados como parcialmente nublados, como el mostrado en la figura 12, sobre las primeras horas del día, es del orden del $60 \%$, disminuyendo a lo largo del día y haciéndose mínima sobre las 15:00 horas. De igual forma, para el día caracterizado como claro, con alta incidencia de radiación, la eficiencia $\eta$ en las primeras horas del día es superior al $90 \%$, como muestra la figura 13.
Todos estos resultados, presentados en las figuras 11,12 y 13 , revelan que en las primeras horas de irradiación (entre las 6:00 y 8:00 horas) se obtienen los valores más elevados de eficiencia, con promedio del orden del $51 \%$, lo que se interpreta como el tiempo en el que el fluido (que se encuentra a la más baja temperatura del ciclo, cercana a los $5{ }^{\circ} \mathrm{C}$ como se puede evidenciar en las figuras 5,6 y 7 ), recibe por transferencia de energía de la placa del colector, la mayor cantidad de energía captada de radiación solar, con la

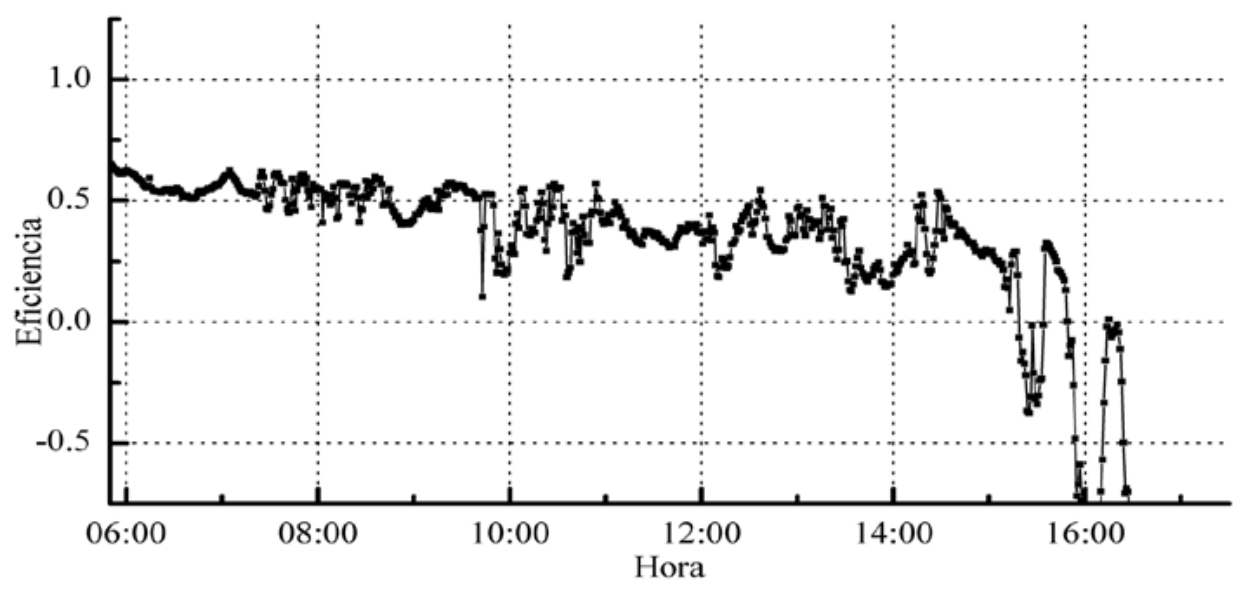

Figura 12. Comportamiento de la eficiencia $\eta$ para el día caracterizado como parcialmente nublado

Fuente: elaboración propia.

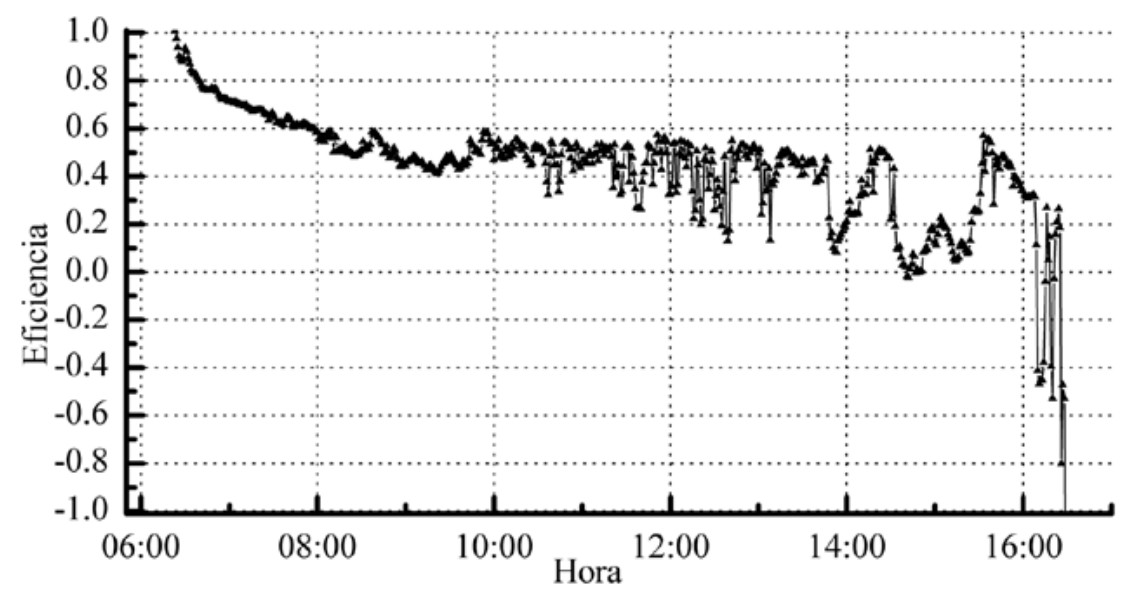

Figura 13. Comportamiento de la eficiencia $\eta$ para el día caracterizado como claro

Fuente: elaboración propia. 
que incrementa su temperatura. Es notable que, posterior a esta franja de tiempo, la eficiencia se mantiene prácticamente constante durante un amplio intervalo de horas, con un promedio cercano al $32 \%$, aunque la temperatura del fluido mantiene una taza de incremento hasta horas del mediodía en todos los casos, tiempo en que se alcanzan las más altas temperaturas del fluido y en el que su promedio es de $36,1{ }^{\circ} \mathrm{C}$. Luego de las horas del mediodía, la eficiencia en promedio se reduce al orden del $26 \%$, hasta llegar sobre las 16:00 horas, en el que se destaca el hecho de que la eficiencia se reduce drásticamente, inclusive obteniendo valores negativos, ya que el fluido comienza el proceso de enfriamiento, como consecuencia de la reducción en la intensidad de la radiación solar.

La figura 14 muestra el promedio diario de eficiencia del colector solar para un periodo de 15 días consecutivos, en los que se obtiene una eficiencia promedio del 36,6\%.

Análogamente a los procesos descritos, se obtienen perfiles diarios de radiación solar global, temperatura ambiente, y las correlaciones correspondientes de eficiencia respecto a las temperaturas de entrada y salida del fluido, temperatura reducida del sistema cada día.

\section{CONCLUSIONES}

Un logro fundamental de este trabajo es el diseño y construcción de un instrumento virtual como arreglo experimental, para el monitoreo del desempeño de colectores solares de placa plana, a partir de sistemas de adquisición de datos de radiación solar global, temperatura ambiente, la temperatura de entrada y salida del fluido, en tiempo real, con el que se determina la temperatura reducida del sistema y la eficiencia instantánea.

Se ha hecho seguimiento del desempeño para días caracterizados en tres intervalos, de baja, media y alta radiación solar global, obteniéndose un promedio diario del 36,6\%, para los días analizados. Adicionalmente, se observa que el desempeño del dispositivo es óptimo, para días en los que el comportamiento climático, es estable, esto es, días nublados o claros, pero como era de esperarse, obteniéndose las más altas temperaturas del fluido, en días claros, inclusive superiores a $60^{\circ} \mathrm{C}$.

Para días claros, el aumento de radiación solar genera incremento de la eficiencia instantánea del colector solar, dado que, bajo estas condiciones, la temperatura reducida del sistema $T_{R}$ disminuye tendiendo a cero. De esta forma se determina la eficiencia óptica del sistema que como resultado

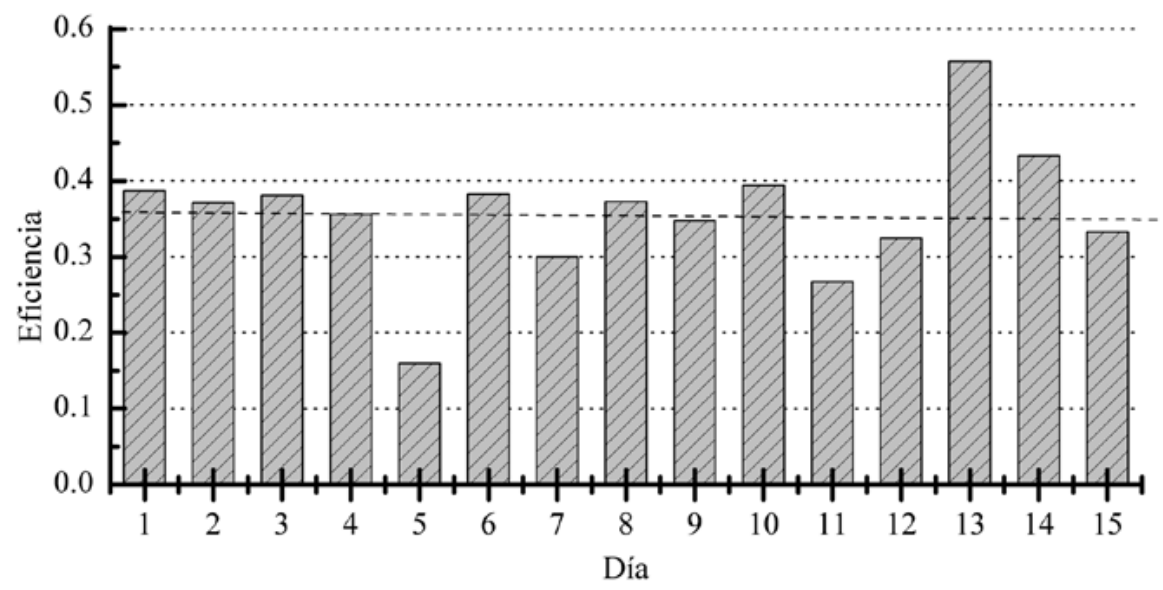

Figura 14. Promedios diarios de eficiencia de un colector solar de placa plana

Fuente: elaboración propia. 
se obtiene del $69,2 \%$, parámetro de referencia de la eficiencia del colector solar de placa plana.

\section{FINANCIAMIENTO}

El Centro de Investigaciones y Desarrollo Científico de la Universidad Distrital Francisco José de Caldas (Bogotá Colombia) financió el proyecto de investigación "Desarrollo y prueba de prototipo de estación de medición de señales que dan información de variables ambientales", a partir del cual se adquirió el software LabVIEW, los módulos Compac FieldPoint y el sensor de radiación solar, con los que se desarrollaron originalmente los sistemas de adquisición de datos.

\section{AGRADECIMIENTOS}

Al Centro de Investigaciones y Desarrollo Científico de la Universidad Distrital Francisco José de Caldas. Igualmente, al ingeniero Fabio L. Roa, encargado del taller de mecánica fina de la Facultad de Ciencias y Educación, por el diseño del montaje experimental.

\section{REFERENCIAS}

Álvarez, A., Cabeza, O., Muñiz, M. y Valera, L. M. (2010). Experimental and numerical investigation of a flat-plate solar collector. Energy, 35(9), 3707-3716. https://doi.org/10.1016/j.energy.2010.05.016

American Society of Heating, Refrigerating and Air-Conditioning Engineers (ASHRAE) (2003). Methods of testing to determine the thermal performance of solar collectors (ASHRAE Standard). Atlanta.

Ayompe, L. y Duffy, A. (2013). Analysis of the thermal performance of a solar water heating system with flat plate collectors in a temperate climate. Applied Thermal Engineering, (58), 447-454. https://doi.org/10.1016/j.applthermaleng.2013.04.062
Devrim, A., Zafer, U. y Olcay, K. (2015). Thermal performance analysis of a solar energy sourced latent. Renewable and Sustainable Energy Reviews, 50, 1213-1225. https://doi.org/10.1016/j. rser.2015.04.195

Duffie, J. y Beckman, W. (2013). Solar engineering of thermal processes. 4a. ed. Nueva Jersey, EE. UU.: John Wiley \& Sons, Inc. https://doi. org/10.1002/9781118671603

Esen, M. y Esen, H. (2005). Experimental investigation of a two-phase closed thermosyphon solar water heater. Solar Energy, 79, 459-468. https://doi.org/10.1016/j.solener.2005.01.001

Forero, N., Caicedo, L. M. y Gordillo, G. (2007). Correlation of global solar radiation values estimated and measured on an inclined surface for clear days in Bogotá. Renewable Energy, 32, 2590-2602. https:// doi.org/10.1016/j.renene.2006.12.012

Liqun, Z., Yiping, W. y Qunwu, H. (2019). Parametric analysis on the performance of flat plate collector with transparent insulation material. Energy, 174, 534-542. https://doi.org/10.1016/j. energy.2019.02.168

Ministerio de Ambiente y Desarrollo Sostenible (MADS) (18 de noviembre de 2019). Impacto del cambio climático en Colombia. Recuperado de http:// www.minambiente.gov.co/index.php/component/ content/article/457-plantilla-cambio-climatico-13

Saedodin, S., Zamzamian, S. A. H., Nimvari, M. E., Wongwises, S. y Jouybari, H. J. (2017). Performance evaluation of a flat-plate solar collector filled with porous metal foam: Experimental and numerical analysis. Energy Conversion and Management, 153, 278-287. https://doi.org/10.1016/j. enconman.2017.09.072

Vestlund, J., Dalenba, J.-O. y Ronnelid, M. (2012). Thermal and mechanical performance of sealed, gas-filled, flat plate solar collectors. Solar Energy, 86, 13-25. https://doi.org/10.1016/j. solener.2011.08.023

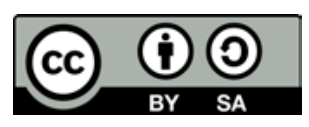

Tecnura • p-ISSN: 0123-921X • e-ISSN: 2248-7638 • Vol. 24 No. 65 • Julio - Septiembre de $2020 \bullet$ pp. 109-124 